\title{
Korean clinical practice guidelines for preventing transmission of coronavirus disease 2019 (COVID-19) in hemodialysis facilities
}

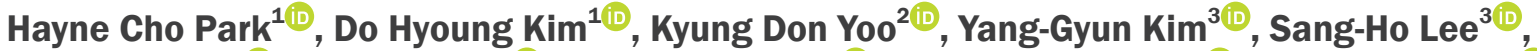

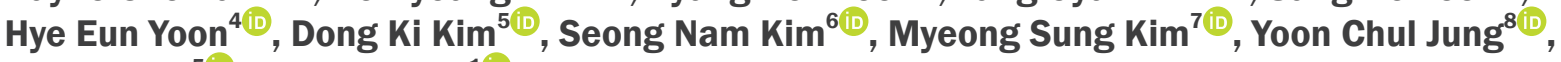 \\ Yon Su Kim ${ }^{5}$, Young-Ki Lee ${ }^{1 \mathbb{D}}$; The Korean Society of Nephrology COVID-19 Task Force Team \\ 'Department of Internal Medicine, Hallym University College of Medicine, Seoul, Republic of Korea \\ ${ }^{2}$ Division of Nephrology, Department of Internal Medicine, Ulsan University Hospital, University of Ulsan College of Medicine, Ulsan, \\ Republic of Korea \\ ${ }^{3}$ Department of Internal Medicine, Kyung Hee University College of Medicine, Seoul, Republic of Korea \\ ${ }^{4}$ Depatment of Internal Medicine, The Catholic University of Korea College of Medicine, Seoul, Republic of Korea \\ ${ }^{5}$ Department of Internal Medicine, Seoul National University College of Medicine, Seoul, Republic of Korea \\ ${ }^{6}$ Kim Seong Nam Internal Medicine Clinic, Seoul, Republic of Korea \\ ${ }^{7}$ Gojan Myeong Internal Medicine Clinic, Ansan, Republic of Korea \\ ${ }^{8}$ Department of Internal Medicine, Bundang Jesaeng General Hospital, Seongnam, Republic of Korea
}

Coronavirus disease 2019 (COVID-19) is a highly contagious viral disease that is caused by the novel virus Severe Acute Respiratory Syndrome Coronavirus-2 (SARS-CoV-2). COVID-19 has become pandemic since December 2019, when the first case developed in Wuhan, China. Patients receiving hemodialysis are more vulnerable to viral transmission because their immune functions are impaired and they receive treatment within a narrow space. Calling on previous experience with Middle East Respiratory Syndrome during the 2015 outbreak, the joint committee of the Korean Society of Nephrology and the Korean Society of Dialysis Therapy quickly formed a COVID-19 task force team to develop a manual before the first index case was diagnosed in the hemodialysis unit. This special article introduces clinical practice guidelines to prevent secondary transmission of COVID-19 within hemodialysis facilities, which were developed to protect patients, healthcare workers, and caregivers from this highly transmissible virus. The areas of infection control covered by these guidelines include standard precautions, performing dialysis therapy for confirmed or suspected cases, performing cohort isolation for contact patients, and disease monitoring and contact surveillance. We hope these guidelines help healthcare workers and hemodialysis patients around the world cope with the COVID-19 pandemic.

Keywords: COVID-19, Hemodialysis units, Infection control, Practice guideline

Received March 31, 2020; Revised April 5, 2020; Accepted April 7, 2020

Edited by Gheun-Ho Kim, Hanyang University, Seoul, Republic of Korea

Correspondence: Young-Ki Lee

Department of Internal Medicine, Hallym University Kangnam Sacred Heart Hospital, Hallym University College of Medicine, 1 Singil-ro, Yeongdeungpo-gu, Seoul 07441, Republic of Korea.E-mail:km2071@naver.com

Copyright (C) 2020 by The Korean Society of Nephrology

(a) This is an open-access article distributed under the terms of the Creative Commons Attribution Non-Commercial License (http://creativecommons.org/ licenses/by-nc-nd/4.0/), which permits unrestricted non-commercial use, distribution, and reproduction in any medium, provided the original work is properly cited. 


\section{Introduction}

Coronavirus disease 2019 (COVID-19) has struck the world, with over 1 million people confirmed with the virus and 56,985 attributed deaths as of April 5, 2020 [1]. The causative organism is Severe Acute Respiratory Syndrome Coronavirus-2 (SARS-CoV-2), which belongs to the betacoronavirus genus $[2,3]$. However, compared to previous coronavirus infections such as SARS and Middle East Respiratory Syndrome (MERS), COVID-19 has shown a higher transmission rate and rapid progression over a short duration of time $[4,5]$. Due to the alarming level of spread and severity of disease, the World Health Organization (WHO) has declared COVID-19 a pandemic.

Korea was the one of the earliest countries to experience a large COVID-19 outbreak. Since the first patient was diagnosed as COVID-19 positive on January 20, 2020, 10,237 patients have been diagnosed with the infection through April 5th, 2020 [6]. Among them, 14 patients, 8 healthcare workers, and 2 caregivers were diagnosed with COVID-19 in a hemodialysis (HD) unit. HD patients receive treatment three times a week in a limited space and are particularly vulnerable to infection due to impaired immunity and environmental susceptibility [7]. Our previous experience fighting MERS [8] and developing infection control guidelines for HD facilities [9] was useful as we promptly responded to the COVID-19 outbreak. This special article introduces our effort to prevent further viral transmission within HD facilities and describes key recommendations for clinical practice.

\section{Establishment and activities of the joint committee}

When the first index case was confirmed in Korea on January 20, 2020, the joint committee was established immediately to respond to the outbreak and prevent transmission of the disease among HD facilities. The joint committee was comprised of nephrologists from the Korean Society of Nephrology and the Korean Society of Dialysis Therapy together with the Korea Centers for Disease Control \& Prevention (KCDC). The committee established and announced the first draft of clinical practice guidelines on January 31, 2020. In addition, the committee delivered policies of the KCDC to regional nephrologists and arranged for patient referral and transfer together with government authorities. Owing to the effort of the committee, the nephrologists could reference the manual without frustration when the first case was confirmed in an HD facility on February 19, 2020.

\section{Development and amendment of clinical practice guidelines for HD facilities}

The clinical practice guidelines for HD facilities were developed based on 'COVID-19 response guidelines' from the KCDC [10]. Since the first release on January 31, 2020 , the guidelines have been revised twice as case definitions and response guidelines from the KCDC changed.

Table 1. Key recommendations from COVID-19 clinical practice guidelines for HD facilities

1. Standard, contact, and droplet precautions should be appropriately performed by all patients and healthcare workers in hemodialysis facilities. The waiting room or resting area should be closed, and surgical masks and hand sanitizer should always be available for patients to use before entering the HD unit.

2. All patients are recommended to check their body temperature and respiratory symptoms before coming to an HD facility. If they have a fever $\geq 37.5^{\circ} \mathrm{C}$ or respiratory symptoms such as dry cough, sore throat, or shortness of breath, they should not visit the HD unit without notifying healthcare workers.

3. A patient with a suspected or confirmed COVID-19 case should be transferred to a healthcare facility with an isolation room and portable dialysis machine. A transportation and management plan should be established in consultation with the public health center and infection control division of the hospital.

4. Contacts are defined as HD patients and healthcare workers who have been in contact with a confirmed case. Contacts should monitor body temperature and respiratory symptoms every day. Those without fever or respiratory symptoms should be subjected to self-quarantine and HD with cohort isolation for 14 days from the last exposure. All transport between home and the HD facility should be provided by the disease prevention authority.

5. Inter-hospital transfer is essentially prohibited during an outbreak. If referral to another hospital is inevitable, the attending physician should transfer patients after careful discussion with the regional disease prevention authority and designated hospital. Patient transfer should be performed only when there is solid evidence that the patient is not infected.

CovID-19, coronavirus disease 2019; HD, hemodialysis. 
The committee subsequently announced a second amendment of the guidelines on February 20, 2020 and the third amendment on March 10, 2020 (Supplementary materials, available online; http://www.ksn.or.kr/ rang_board/list.html?num=1622\&code=notice). The key recommendations of the clinical practice guidelines are summarized in Table 1. Fig. 1 depicts the flowchart for patient screening and management for COVID-19 in HD units.

\section{Case definitions}

Case definitions are from the 'COVID-19 response guidelines' of the KCDC, with cases classified as confirmed case, suspected case, patient under investigation, and contacts (Table 2). A confirmed case refers to a person who has tested positive for the COVID-19 pathogen irrespective of clinical signs and symptoms. A suspected case refers to a person who develops a fever $\geq 37.5^{\circ} \mathrm{C}$ or respiratory symptoms within 14 days after contact with a confirmed patient. A patient under investigation refers to a suspected case based on a physician's opinion or one who develops a fever $\geq 37.5^{\circ} \mathrm{C}$ or respiratory symptoms within 14 days of travelling to a region or country with local transmission of COVID-19. Lastly, contacts within HD facilities are determined after epidemiological investigation by the central and local government under the guidance of the KCDC and are defined in more detail as one of the following: 1) a patient who received HD treatment at the same time as a confirmed case, 2) a patient who received HD treatment without proper disinfection after HD of a confirmed case, or 3) a patient or healthcare worker who was exposed to a confirmed case within 2 meters.

\section{General principles}

All medical staffs, HD patients, and their caregivers should be educated about personal hygiene, including washing hands, wearing masks, and avoiding unnecessary contact. Surgical masks and hand sanitizer should be available at the entrance of the HD unit. Healthcare workers, HD patients, and their caregivers should perform standard, contact, and droplet precautions, including hand hygiene and wearing masks. Each HD unit should prepare an environment suited to minimize infection



Figure 1. Flowchart for patient screening and management of coronavirus disease 2019 (COVID-19) in hemodialysis (HD) units. HD patients should be classified as confirmed case, suspected case, contact, or patient under investigation according to the history of contact with a confirmed case and COVID-19 related symptoms (fever $\geq 37.5^{\circ} \mathrm{C}$ or respiratory symptoms such as cough, sore throat, or shortness of breath). Patients should only be released from quarantine if all related symptoms improve and a COVID-19 test is negative. 
Table 2. Case definition

Confirmed case
$\begin{aligned} & \text { A person who has tested positive for the COVID-19 pathogen in accordance with testing standards } \\ & \text { signs and symptoms }\end{aligned}$
A person who develops a fever $\geq 37.5^{\circ} \mathrm{C}$ or respiratory symptoms such as coughing or difficulty breathing within 14 days of
coming into contact with a confirmed case while that patient was symptomatic
Any of the following:
(1) A person who is suspected of having COVID-19 by their physician (e.g., pneumonia of unknown cause)
(2) A person who develops a fever $\geq 37.5^{\circ} \mathrm{C}$ or respiratory symptoms such as coughing or difficulty breathing within 14
days of travelling to a country with local transmission ${ }^{\mathrm{b}}$ of COVID-19 such as China (including Hong Kong and Macau)
(3) A person with an epidemiological link to the collective outbreak of COVID-19 in Korea who develops a fever $\geq 37.5^{\circ} \mathrm{C}$
or respiratory symptoms such as coughing or difficulty breathing within 14 days
Contacts are determined by epidemiological investigation and include the following:
(1) A patient who received HD treatment at the same time as a confirmed case
(2) A patient who received HD treatment without proper disinfection after HD of a confirmed case
(3) A patient or healthcare worker who was exposed to a confirmed case within 2 meters

COVID-19, coronavirus disease 2019; HD, hemodialysis.

${ }^{a}$ Testing and screening: Severe Acute Respiratory Syndrome Coronavirus-2 (SARS-CoV-2) genetic testing (PCR) or virus separation. ${ }^{b}$ Refer to the World Health Organization (WHO) website (local transmission) or the Korea Centers for Disease Control \& Prevention (KCDC) website (http://ncov.mohw.go.kr/en).

transmission, such as keeping proper distance between beds. The waiting room or resting area should be closed and each HD patient should come in at a reserved time to minimize patient-to-patient contact. Before arrival at the HD unit, all HD patients should be asked about their prior visit to other countries and domestic outbreak regions, any patient contact history, and their clinical symptoms.

If a patient is classified as a suspected case or patient under investigation, they should contact the local public health center or KCDC call center ( 8 area number+120 or 81339 ) and follow the instructions. In addition, if the patient has a fever, cough, or shortness of breath, they should call first and let the healthcare workers know about their symptoms before they visit the HD unit. All HD patients should enter the HD unit under the permission of the physician only if they do not have suspicious signs or symptoms of COVID-19. It is most important that suspected or confirmed COVID-19 patients do not enter the HD unit. Each HD unit should also prevent secondary transmission of infection by restricting caregivers or visitors in the HD unit.

Lastly, inter-hospital transfer should be essentially prohibited during the outbreak unless the transfer of the patient to the other institution has no risk of infection transmission. If a confirmed or suspected case should be transferred to another hospital, the HD unit should consult in advance with the regional public health center and the hospital that will receive the patient.

\section{Response measures for suspected cases}

When a suspected case visits the HD unit, the patient should wear a surgical mask and be isolated temporarily until a decision can be made about their care. If the HD unit has the capacity of performing dialysis therapy in a negative-pressure isolation room with a portable dialysis machine, the HD unit may perform isolation dialysis therapy until the patient is released from quarantine. However, if the HD unit cannot accommodate isolation dialysis, as is most often the case, the patient should be transferred to another hospital with a facility for isolation dialysis. Each HD unit should establish a transfer plan in consultation with the regional public health center or the infection control department and should secure a separate route to evacuate the patient from the HD unit. Transportation between the HD unit and the referral hospital should be determined by the regional public health center. After transfer of the suspected case, the HD room and other spaces where the patient stayed should be appropriately disinfected immediately.

\section{Infection control measures when performing HD therapy for a confirmed/suspected case}

When a patient is confirmed or suspected to have COVID-19, they should be isolated in a single room with negative pressure. The HD patient should receive HD therapy regularly and separately from other uninfected HD pa- 
tients. Therefore, the confirmed/suspected case should receive HD therapy in an isolated room with a portable dialysis machine. Healthcare workers who perform the HD therapy should wear personal protective equipment (PPE) that includes a mask (KF94 or N95 respirator mask with a highly efficient filter function), gloves, goggles or facial shield, and gown (Level D). Healthcare workers should perform hand hygiene before and after contacting the patient and after removing PPE. The dialysis machine should be disinfected after use according to the manufacturer's recommendations. The room should be properly cleaned and disinfected after each dialysis session and should be emptied for a few hours before the next use. All medical waste should be disposed of according to relevant guidelines. The confirmed/suspected case can be released from quarantine if symptoms improve and tests for COVID-19 are negative twice with a 24-hour interval. If respiratory symptoms later become aggravated, a confirmatory test can be repeated until symptoms improve.

\section{Response measures for patients under investigation}

Patients under investigation must not enter the HD unit but should contact the local public health center or KCDC call center (\&area number +120 or $\mathbf{8} 1339$ ) and visit a screening center to receive a COVID-19 test. If such a patient has already visited the HD unit, healthcare workers should secure an evacuation route to ensure the patient does not contact other patients. The patient under investigation should not receive HD therapy until COVID-19 test results turn out negative. The patient should receive HD therapy apart from other patients after the test turns negative. If respiratory symptoms improve and tests are negative for twice with a 24-hour interval, the patient can receive HD therapy together with other patients. However, if symptoms persist, the patient should maintain separate HD therapy, even with a twice negative test.

\section{Response measures for contacts}

Asymptomatic contacts who have had contact with a confirmed or suspected case should receive screening tests for COVID-19 and be referred to the HD with cohort isolation when the test results are negative. An HD with cohort isolation is a method of dialysis therapy provided to asymptomatic contacts in the affected hos- pital at a different time apart from other patients. If the contact patient develops fever or respiratory symptoms, they are classified as a suspected case and should follow the respective response measures. Healthcare workers should wear PPE, including masks (KF94 or N95), gloves, goggles or facial shield, and a disposable waterproof gown. During HD therapy, standard, contact, and droplet precautions should be followed. HD with cohort isolation should be maintained for 14 days from the date of contact. All asymptomatic contacts should receive a COVID-19 screening test on the 13th day from the date of contact and may be released from cohort isolation if the tests are negative.

\section{Discussion}

The COVID-19 pandemic has posed a global health threat, affecting over 1 million people globally with thousands of deaths. Patients on HD treatment are more vulnerable to infection due to low immune function and high comorbidities. Since they receive routine treatment, usually three times a week, in the same HD unit, all possible measures should be taken to prevent and eradicate infection transmission there. Recently, a few guidelines from affected countries have been published [5,11-13]. Although they describe general principles to prevent viral transmission in HD units, they are too broad for real world application. The main difference in our Korean guidelines is the classification by transmission risk and associated recommended strategy. The current guidelines direct clinicians and patients to follow measures of prevention, protection, screening, and isolation, and describe the circumstances for release from quarantine. We hope these guidelines play a key role in the prevention of secondary transmission of COVID-19 during this pandemic.

\section{Conflicts of interest}

COVID-19 clinical practice guidelines for HD facilities were developed by a joint committee comprised of members from the Korean Society of Nephrology, the Korean Society of Dialysis Therapy, and the Korean Center for Disease Control \& Prevention. We hereby declare that the authors and members of the Korean Society of Nephrology COVID-19 Task Force participated in the develop- 
ment and amendment of these guidelines but have not received any research grants that could potentially influence their publication.

\section{Acknowledgments}

We would like to thank all the board members from the Korean Society of Nephrology and the Korean Society of Dialysis Therapy for supporting the development and amendment of these COVID-19 clinical practice guidelines for HD facilities. We would also like to offer sincere gratitude to all physicians and nurses, especially those in Daegu and Kyungpook provinces, for their dedicated efforts and time spent treating HD patients within quarantine settings.

\section{Authors' contributions}

Hayne Cho Park participated in the COVID-19 immediate response team and drafted the guideline and manuscript. Do Hyoung Kim, Seong Nam Kim, Myeong Sung Kim, and Yoon Chul Jung participated in the data collection from each hemodialysis units. Kyung Don Yoo, YangGyun Kim, Sang-Ho Lee, and Hye Eun Yoon participated in the COVID-19 immediate response team and revised the manuscript. Dong Ki Kim and Yon Su Kim provided intellectual content of critical importance to the work and technical support. Young-Ki Lee participated in the study design and coordination, led the COVID-19 Task Force Team and helped to draft and revise the manuscript. All authors read and approved the final manuscript.

\section{References}

[1] World Health Organization. Coronavirus disease (COVID-2019) situation reports [Internet]. Geneva (Switzerland): World Health Organization, c2020 [cited 2020 Apr 5]. Available from: https://www.who.int/emergencies/diseases/ novel-coronavirus-2019/situation-reports.

[2] Lu R, Zhao X, Li J, et al. Genomic characterisation and epidemiology of 2019 novel coronavirus: implications for virus origins and receptor binding. Lancet 2020;395:565-574.

[3] Kim JM, Chung YS, Jo HJ, et al. Identification of Coronavirus isolated from a patient in Korea with COVID-19. Osong Public Health Res Perspect 2020;11:3-7.
[4] Mahase E. Coronavirus COVID-19 has killed more people than SARS and MERS combined, despite lower case fatality rate. BMJ 2020;368:m641.

[5] Naicker S, Yang CW, Hwang SJ, Liu BC, Chen JH, Jha V. The novel Coronavirus 2019 epidemic and kidneys. Kidney Int 2020;97:824-828.

[6] Korea Centers for Disease Control \& Prevention. Cases of COVID-19 in Korea [Internet]. Cheongju (Korea): Korea Centers for Disease Control \& Prevention, 2020 [cited 2020 Apr 4]. Available from: http://ncov.mohw.go.kr/en/ bdBoardList.do?brdId=16\&brdGubun $=161 \&$ dataGubun $=\&$ ncvContSeq=\&contSeq=\&board_id.

[7] Park HC, Lee SH, Kim J, et al. Effect of isolation practice on the transmission of Middle East respiratory syndrome Coronavirus among hemodialysis patients: a 2-year prospective cohort study. Medicine (Baltimore) 2020;99:e18782.

[8] Park HC, Lee YK, Lee SH, et al.; Korean Society of Nephrology MERS-CoV Task Force Team. Middle East respiratory syndrome clinical practice guideline for hemodialysis facilities. Kidney Res Clin Pract 2017;36:111-116.

[9] Park HC, Lee YK, Yoo KD, et al. Korean clinical practice guidelines for preventing the transmission of infections in hemodialysis facilities. Kidney Res Clin Pract 2018;37:8-19.

[10] Korea Centers for Disease Control \& Prevention. Case definition and people subject to testing [Internet]. Cheongju (Korea): Korea Centers for Disease Control \& Prevention, 2020 [cited 2020 Mar 28]. Available from: http://ncov.mohw. go.kr/en/baroView.do?brdId=11\&brdGubun=112\&dataGub un=\&ncvContSeq=\&contSeq=\&board_id=\&gubun.

[11] Expert team of Chinese Medical Association Nephrology Branch. [Recommendations for prevention and control of novel Coronavirus infection in blood purification center (room) from the Chinese Medical Association Nephrology Branch]. Chin J Nephrol 2020;36:82-84. Chinese.

[12] Centers for Disease Control and Prevention. Recommendations for patients with suspected or confirmed COVID-19 in outpatient hemodialysis facilities [Internet]. Atlanta (USA): Centers for Disease Control and Prevention, 2020 [cited 2020 Mar 30]. Available from: https://www.cdc.gov/ coronavirus/2019-ncov/healthcare-facilities/dialysis.html.

[13] Basile C, Combe C, Pizzarelli F, et al. Recommendations for the prevention, mitigation and containment of the emerging SARS-CoV-2 (COVID-19) pandemic in haemodialysis centres. Nephrol Dial Transplant 2020;35:737-741. 